\title{
A Novel Preamble Design for OFDM Transmission Parameter Signalling
}

\author{
Lifeng He, Zhaocheng Wang and Fang Yang \\ Tsinghua National Laboratory for Information Science \\ and Technology (TNList), Tsinghua University \\ Beijing, 100084, P. R. China \\ Email: hlf04@mails.tsinghua.edu.cn
}

\author{
Sheng Chen and Lajos Hanzo \\ School of Electronics and Computer Science \\ University of Southampton \\ Southampton SO17 1BJ, U.K. \\ Email:sqc@ecs.soton.ac.uk
}

\begin{abstract}
A novel preamble design is proposed for orthogonal frequency division multiplexing systems, which exploits the variable distance between a pair of training sequences for the transmission parameter signalling. Compared to the existing P1-symbol based preamble for the second generation digital terrestrial television broadcasting standard, the proposed design maintains the high performance and robustness in timing and carrier frequency offset estimation while significantly reducing the signalling detection complexity. Simulation results demonstrate that the proposed preamble achieves a better signalling detection performance than the standardized P1 symbol design.
\end{abstract}

\section{INTRODUCTION}

Orthogonal frequency division multiplexing (OFDM) technology [1] has been widely applied in the areas of digital TV [2]-[5], wireless local area networks [1], [6], and the next generation mobile communications [7], [8]. With the growing commercial demands for supporting multi-service broadcast, including HDTV, mobile TV and data-casting, the broadcast system is expected to provide a wide choice of transmission parameters to accommodate with different requirements for quality of service. The European Telecommunications Standards Institute (ETSI) recently issued the second generation digital terrestrial television broadcasting standard (DVB-T2), which aims at providing multi-services with different robustness [5]. DVB-T2 offers a total of six fast Fourier transform (FFT) sizes and seven guard interval (GI) modes for different applications. Furthermore, both singleinput single-output (SISO) and multiple-input single-output (MISO) transmission modes are supported.

Quick and reliable detection of the transmission parameters is critical for the receiver to perform subsequent processing. For this purpose, DVB-T2 adopts a specially designed P1 symbol as the preamble of the DVB-T2 frame. Unlike conventional preambles which are designed merely for timing and frequency synchronization [9], [10], the P1 symbol also transmits the basic transmission parameter signalling (TPS), including the FFT size and SISO/MISO mode [5]. In the time domain, a novel cyclic extension structure is adopted to sharpen the peak of the GI correlation (GIC) for better timing synchronization [11]. In the discrete Fourier transform (DFT) domain, a length384 sequence carrying 7-bit signalling is modulated into the distributed carrier pattern. To transmit the 7-bit signalling, the P1 symbol in DVB-T2 exploits two sets of orthogonal complementary sequences to represent different signalling fields, which are referred to as S1 and S2, respectively. At the receiver, all the possible sequences in the both sets are correlated with the received signalling sequence to find a matched case. Therefore, a large number of correlations are required, which imposes a high computational complexity for signalling detection.

In this contribution, a novel preamble design is proposed, which achieves the same signalling purpose of the P1 symbol based preamble at a lower complexity. Specifically, unlike the P1 symbol which uses different sequences to indicate the signalling, the proposed preamble inserts a pair of training sequences in the DFT domain, and the distance between the pair is utilised for signalling. At the receiver, only a single correlator is required to estimate both the TPS and carrier frequency offset (CFO) simultaneously and, therefore, the complexity of signalling detection is reduced significantly. Furthermore, our simulation results show that the proposed preamble design achieves better signalling performance than the P1-symbol based preamble of the DVB-T2 over frequencyselective fading channels.

\section{P1 Symbol Design In DVB-T2}

The transmitted time-domain signal of an OFDM system can be represented by an inverse FFT (IFFT) as follows

$$
x_{n}=\frac{1}{\sqrt{N}} \sum_{k=0}^{N-1} X_{k} \cdot e^{j \frac{2 \pi}{N} n k}, 0 \leq n \leq N-1,
$$

where $N$ is the number of subcarriers in the OFDM system, and $X_{k}$ are the transmitted data symbols. At the receiver, the received OFDM symbol is represented by

$$
y_{n}=x_{n-n_{0}} \otimes h_{n} e^{j 2 \pi f_{c} n}+\nu_{n}
$$

where the operator $\otimes$ represents linear convolution, $n_{0}$ and $f_{c}$ denote the time delay and CFO, respectively, while $h_{n}$ and $\nu_{n}$ denote the channel impulse response and the additive white Gaussian noise (AWGN), respectively. The channel signal-tonoise ratio (SNR) is defined by $\rho=\sigma_{s}^{2} / \sigma_{n}^{2}$, where $\sigma_{s}^{2}=$ $E\left[\left|x_{n}\right|^{2}\right]$ is the signal power and $\sigma_{n}^{2}=E\left[\left|\nu_{n}\right|^{2}\right]$ is the AWGN power, with $E[\bullet]$ denoting the expectation operator. 

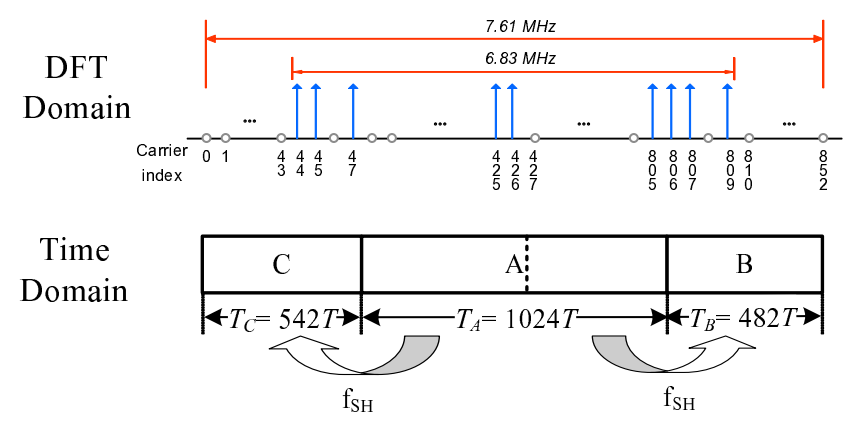

Fig. 1. Structure of the P1 symbol in DVB-T2.

The CFO can be separated into the two parts as follows

$$
f_{c}=m_{\mathrm{int}} \cdot \frac{1}{N}+f_{\text {frc }},
$$

where $\frac{1}{N}$ is the subcarrier spacing, $m_{\text {int }}$ is an integer, and $f_{\text {frc }}$ is the fractional part of the CFO in the range of

$$
-\frac{1}{2 N}<f_{\text {frc }} \leq \frac{1}{2 N}
$$

The integer part of CFO will lead to the carrier shift in the DFT domain, while the fractional CFO will cause inter-carrier interference, which can severely degrade the performance of OFDM demodulation [12]. In OFDM systems, training symbols are often transmitted before the data blocks as preambles, which are used to accomplish timing and frequency synchronization as well as to detect the TPS if it is present.

\section{A. Structure of the P1 Symbol}

The P1 symbol defined in the DVB-T2 standard [5] consists of a $1 \mathrm{~K}$ OFDM symbol ' $\mathrm{A}$ ' and its two cyclic extensions, denoted as ' $\mathrm{C}$ ' and 'B', respectively. The C-A-B structure of the $\mathrm{P} 1$ symbol is illustrated in Fig. 1. The time-domain signal of the P1 symbol is defined by

$$
x_{n}= \begin{cases}p_{n} \cdot e^{j 2 \pi f_{\mathrm{SH}} n}, & 0 \leq n<542, \\ p_{n-542}, & 542 \leq n<1566, \\ p_{n-1024} \cdot e^{j 2 \pi f_{\mathrm{SH}} n}, & 1566 \leq n<2048,\end{cases}
$$

where the sequence $\left\{p_{n}\right\}$ is the baseband representation of the part ' $\mathrm{A}$ ', $f_{\mathrm{SH}}$ is the additional frequency shift applied to both the parts ' $\mathrm{B}$ ' and ' $\mathrm{C}$ ' in order to distinguish the $\mathrm{P} 1$ symbol from common cyclic prefix OFDM symbols.

In the DFT domain, out of the 853 useful carriers of the $1 \mathrm{~K}$ symbol in ' $\mathrm{A}$ ', only 384 sub-carriers are used, leaving others set to zeros. The carrier distribution of the P1 symbol is also illustrated in Fig. 1. The active carriers occupy roughly $6.83 \mathrm{MHz}$ in the middle of the nominal $7.61 \mathrm{MHz}$ bandwidth. Even a frequency shift up to $500 \mathrm{kHz}$ can be estimated since most of the useful sub-carriers are still within the bandwidth. Therefore, the P1 symbol is robust to large CFOs.

The P1 signalling contains two fields, known as the 3-bit signalling S1 and the 4-bit signalling S2, respectively. Specifically, $\mathrm{S} 1$ is encoded by one of the 8 orthogonal complementary sequences of length 64 , while S2 is encoded by one of the 16 orthogonal complementary sequences of length 256 [5].
The S1 sequence, the S2 sequence and a repetition of the S1 sequence are concatenated together to compose a length384 signalling sequence, which is firstly differentially binary phase-shift keying (DBPSK) modulated, then scrambled, and afterwards mapped into the active carriers. It should be noted that a total of 128 different signalling sequences correspond to the 7-bit signalling.

\section{B. Detection of the P1 Symbol}

In the receiver, timing synchronization and fractional $\mathrm{CFO}$ estimation can be achieved by a modified GIC method based on the C-A-B structure [11]. After the fractional CFO is compensated, the part 'A' of the P1 symbol is extracted and transformed to the DFT domain for integer CFO estimation and signalling detection. First, the sub-carrier pattern matching is performed to locate the exact positions of the active carriers by correlating the power distribution of the received P1 symbol across the carriers with the expected carrier-distribution sequence (CDS) [13]. The CDS is carefully designed such that only the perfect match with the distribution gives a peak of the power correlation. The position of the correlation peak also gives an estimate of the integer $\mathrm{CFO}$, which is equal to the peak's shift from its designed position.

Once the active carriers are located, the receiver is ready to detect S1 and S2 signalling. The length-384 signalling sequence is firstly extracted from the active carriers, then unscrambled and differentially decoded. Finally, the S1 and S2 fields are separated from the signalling sequence. Each legitimate sequence of the $\mathrm{S} 1$ set is correlated with the received S1 signalling sequence one by one, and the sequence with the largest correlation peak is picked up to decode the S1 signalling. Similarly, each legitimate sequence of the S2 set is correlated with the received S2 signalling sequence one by one to decode the S2 signalling. Since there are totally 8 sequences in the $\mathrm{S} 1$ set and 16 sequences in the S2 set, a large number of correlations are required which leads to a high complexity in detecting the TPS.

\section{The Proposed Preamble Design}

\section{A. Structure of the Proposed Preamble}

The structure of the proposed preamble is shown in Fig. 2. The proposed preamble inherits the identical structure to the existing P1 symbol in the time domain, while in the DFT domain, the TPS design is very different from that of the P1 symbol. Specifically, a pair of identical DBPSK modulated training sequences, denoted by $a$, are inserted into the $N$ subcarriers. The DFT values $\left\{X_{k}\right\}_{k=0}^{N-1}$ of the part 'A' in the

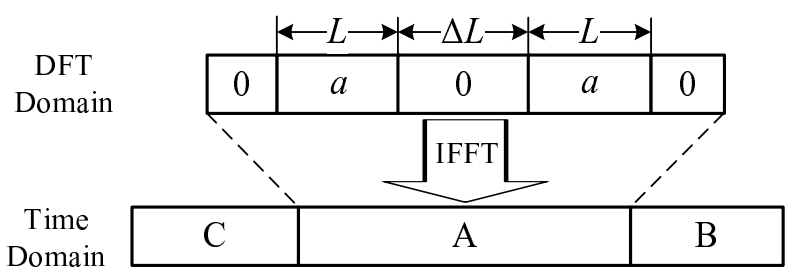

Fig. 2. Structure of the proposed preamble. 
proposed preamble are represented by

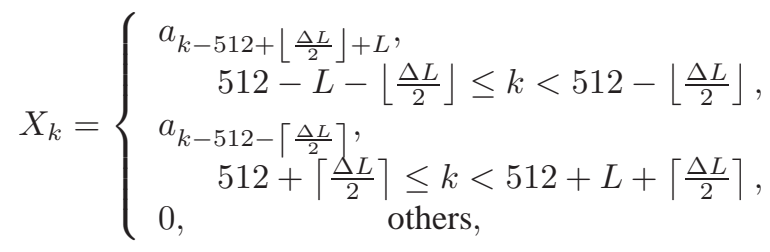

where $\lceil\bullet\rceil$ and $\lfloor\bullet\rfloor$ denote the integer ceiling and floor operators, respectively, $L$ is the length of $a$, while $\Delta L$ is the distance between the pair, which varies according to the TPS need. $\left\{X_{k}\right\}_{k=0}^{N-1}$ are then converted to the time-domain part 'A' of the preamble by the $N$-point IFFT.

The proposed preamble adopts the distance between a pair of training sequences for signalling, and the distance can vary in a wide range of values to accommodate the signalling needs. Taking a pair of length-255 training sequences, for example, the pair occupies two consecutive segments of 255 sub-carriers which are allocated in symmetry from the centre of the nominal bandwidth, as seen in Fig. 2. $\Delta L$ is chosen to vary in the range of $[128,255]$ so that 7-bit signalling can be encoded into the 128 distance values. The carriers at the both ends are reserved so that the preamble can cope with large CFOs, and the carriers in the centre of the bandwidth are also reserved to reduce the impact of carrier leakage.

\section{B. Detection of the Proposed Preamble}

In the receiver, the same GIC method given in [11] can be applied for timing synchronization and fractional $\mathrm{CFO}$ estimation. Since the P1 symbol and proposed preambles have identical time-domain structure, a similar performance in timing and fractional CFO estimation can be expected for the both designs. However, the proposed preamble enables a simpler signalling detection. After the fractional CFO compensation, the part ' $A$ ' is extracted from the preamble and converted to the DFT domain by the $N$-point FFT operation

$$
Y_{k}=\frac{1}{\sqrt{N}} \sum_{n=0}^{N-1}\left(y_{n} e^{-j 2 \pi \hat{f}_{\text {frc }} n}\right) e^{-j \frac{2 \pi}{N} n k}, 0 \leq k<N
$$

where $\hat{f}_{\text {frc }}$ is the estimated fractional CFO. $\left\{Y_{k}\right\}_{k=0}^{N-1}$ is firstly differentially decoded, and then correlated with the local training sequence to yield

$$
R_{k}=\frac{\sum_{l=0}^{L-1}\left(Y_{(l+k) \bmod N} \cdot Y_{(l+k-1) \bmod N}^{*}\right) \cdot\left(a_{l} \cdot a_{l-1}^{*}\right)^{*}}{\frac{1}{2} \sum_{l=0}^{N-1}\left|Y_{l}\right|^{2}},
$$

where $0 \leq n<N,{ }^{*}$ and $\bullet \bmod \bullet$ denote the complex conjugation and modular operators, respectively.

Since there are two identical training sequences in $\left\{Y_{k}\right\}$, two peaks are expected in the correlation (8), and the distance between the two peaks gives an estimation of $\Delta L$ as

$$
\Delta \hat{L}=k_{2}-k_{1}-L
$$

where $k_{1}$ and $k_{2}$ are the peak positions in the first half and the second half of $\left\{R_{k}\right\}$, respectively, given by

$$
\begin{aligned}
& k_{1}=\arg \max _{0 \leq k<\frac{N}{2}}\left|R_{k}\right|, \\
& k_{2}=\arg \max _{\frac{N}{2} \leq k<N}\left|R_{k}\right| .
\end{aligned}
$$

$\Delta \hat{L}$ is then used to decode the 7-bit signalling. Simultaneously, the peak position also yields an estimate of the integer CFO, which is obtained from the peaks' shifts from their designed positions according to

$\hat{m}_{\text {int }}= \begin{cases}\left.k_{1}-\left(512-\mid \frac{\Delta L+L}{2}\right\rfloor\right), & \left|R_{k_{1}}\right| \geq\left|R_{k_{2}}\right|, \\ \left.k_{2}-\left(512+\mid \frac{\Delta L+L}{2}\right\rceil\right), & \left|R_{k_{1}}\right|<\left|R_{k_{2}}\right| .\end{cases}$

In contrast to the P1 symbol design, only a single correlator is required for detecting the TPS, yielding a dramatic reduction in the receiver complexity.

An example of the correlation (8) in the AWGN channel given $\mathrm{SNR}=0 \mathrm{~dB}$ is shown in Fig. 3 . We can observe a shift of the peak position when $\mathrm{CFO}=500 \mathrm{kHz}$ from the reference position of $\mathrm{CFO}=0$. This shift gives a fine estimation of the integer CFO. It can also be seen from Fig. 3 that an accurate estimate of $\Delta L$ can be obtained from the distance between the two correlation peaks.

\section{PERFormance Evaluation}

\section{A. Theoretical Lower Bound over AWGN Channel}

We adopt the method similar to the one given in [9] to analyse the performance of the proposed preamble in the AWGN channel. In the AWGN case, the real part of the correlation peaks, $R_{k_{1}}$ and $R_{k_{2}}$, are the Gaussian distributed random variables with the expectation and variance given respectively by

$$
\mu_{R}=\frac{\rho}{\rho+1}
$$

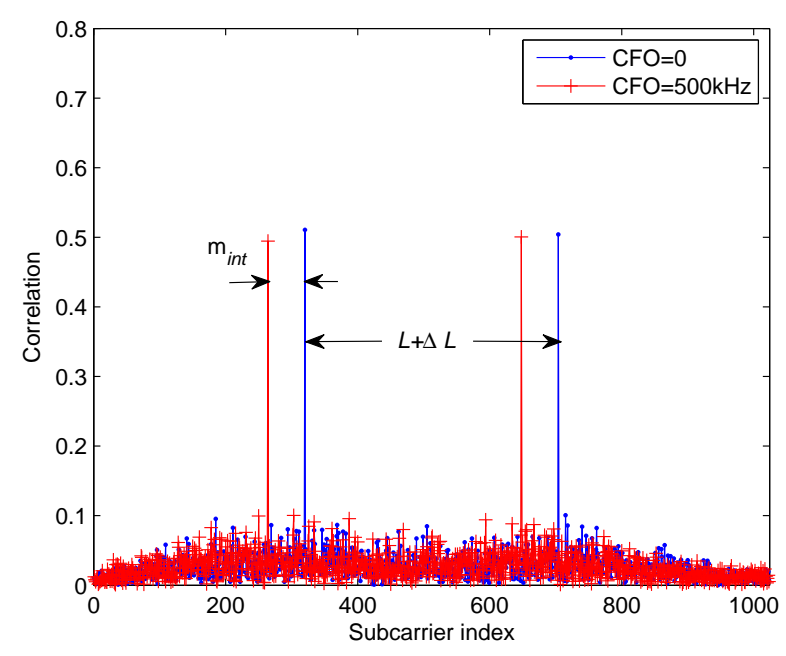

Fig. 3. Correlation results of the proposed preamble design in the AWGN channel given $\mathrm{SNR}=0 \mathrm{~dB}$. 


$$
\sigma_{R}^{2}=\frac{\left(1+\mu_{R}^{2}\right) \rho+\left(2 L / N+\mu_{R}^{2}\right)}{N(\rho+1)^{2}} .
$$

And the imaginary parts of $R_{k_{1}}$ and $R_{k_{2}}$ follow $N\left(0, \sigma_{R}^{2}\right)$ Thus, the peak metrics, $\left|R_{k_{1}}\right|$ and $\left|R_{k_{2}}\right|$, follow the same Rician distribution given by

$$
f_{\text {peak }}(y)=\frac{y}{\sigma_{R}^{2}} e^{-\frac{y^{2}+\mu_{R}^{2}}{2 \sigma_{R}^{2}}} I_{0}\left(\frac{\mu_{R} \cdot y}{\sigma_{R}^{2}}\right), y>0,
$$

where $I_{0}(\bullet)$ is the zero-order modified Bessel function of the first kind [15]. Assuming the ideal correlation property of the training sequences, both real and imaginary parts of the side-lobes are zero-mean Gaussian distributed random variables with the variance of $\sigma_{R}^{2}$. Hence, the side-lobe metric $\left|R_{k}\right|_{k \neq k_{1}, k_{2}}$ has the Rayleigh distribution given by

$$
f_{\text {side }}(y)=\frac{y}{\sigma_{R}^{2}} e^{-\frac{y^{2}}{2 \sigma_{R}^{2}}}, y>0 .
$$

The expectations of the correlation peak and side-lobes given different SNR values are illustrated in Fig. 4, where the dashed lines indicate the standard deviations from the expectation. We can observe that for $\mathrm{SNR} \geq-5 \mathrm{~dB}$, the correlation metric can reliably detect the peak.

Next the probability of false detection is analysed. Considering the first half of $\left\{R_{k}\right\}$, the probability that the side-lobe $\left|R_{k}\right|_{k \neq k_{1}}$ is larger than $\left|R_{k_{1}}\right|$ is given by

$P\left(\left|R_{k}\right|_{k \neq k_{1}}>\left|R_{k_{1}}\right|\right)=\int_{0}^{+\infty} \frac{y}{\sigma_{R}^{2}} e^{-\frac{2 y^{2}+\mu_{R}^{2}}{2 \sigma_{R}^{2}}} I_{0}\left(\frac{\mu_{R} \cdot y}{\sigma_{R}^{2}}\right) d y$.

The false peak detection probability is therefore given by

$$
\begin{aligned}
P_{f} & =P\left(\left\{\max _{0 \leq k<\frac{N}{2}, k \neq k_{1}}\left|R_{k}\right|\right\}>\left|R_{k_{1}}\right|\right) \\
& =1-\left(1-P\left(\left|R_{k}\right|_{k \neq k_{1}}>\left|R_{k_{1}}\right|\right)\right)^{\frac{N}{2}-1} .
\end{aligned}
$$

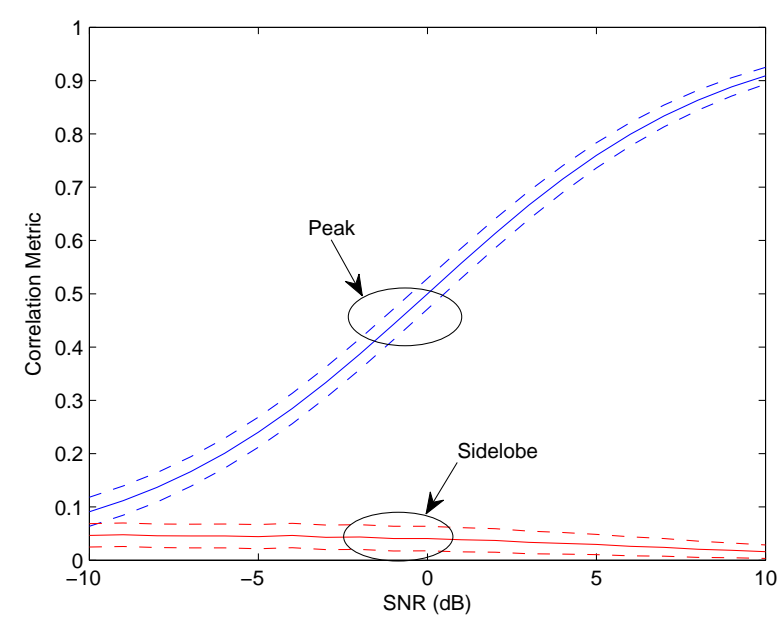

Fig. 4. Expectations of the correlation metric for the proposed preamble design in the AWGN channel given different SNR values, where the dashed lines indicate the standard deviations from the expectation.

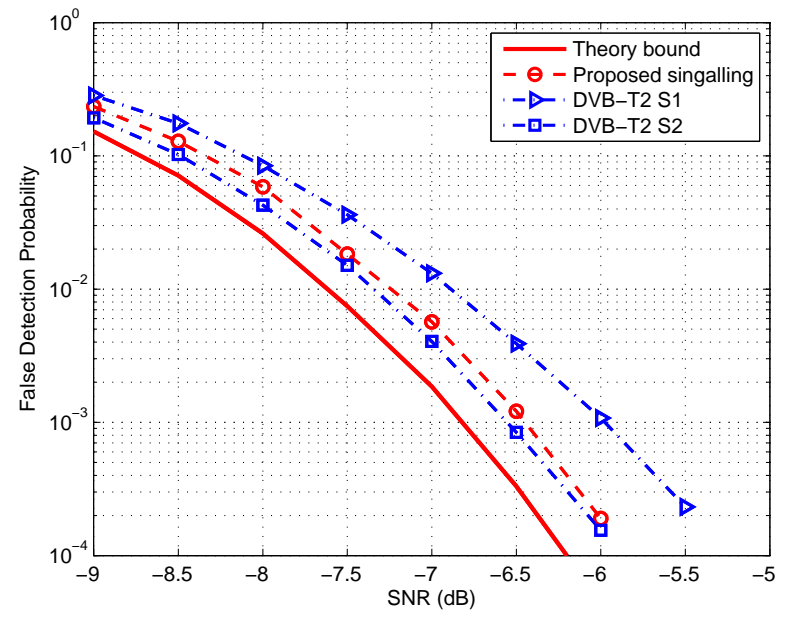

Fig. 5. False detection probability of the proposed signalling detection over the AWGN channel.

Only when the both peaks are detected, the estimation of $\Delta L$ and $m_{\text {int }}$ can be achieved. Thus the false detection probability for $\Delta L$, denoted as $P_{\mathrm{FD}, \Delta L}$, and the false detection probability for $m_{\mathrm{int}}$, denoted as $P_{\mathrm{FD}, m_{\mathrm{int}}}$, are given by

$$
P_{\mathrm{FD}, \Delta L}=P_{\mathrm{FD}, m_{\mathrm{int}}}=1-\left(1-P_{f}\right)^{2} .
$$

$P_{\mathrm{FD}, \Delta L}$ or $P_{\mathrm{FD}, m_{\mathrm{int}}}$ is the false detection probability of the correlation detector, which is the function of SNR.

In order to guarantee that the receiver works properly, the signalling has to be detected with no error. Therefore, signalling error rate (SER), defined as the false detection probability, was used to evaluate the performance of the signalling detection. Fig. 5 depicts the signalling detection performance of the proposed preamble in the AWGN channel. The solid curve in Fig. 5 gives the theory lower bound through a numerical computation of (17) to (19). However, for the practical system which has imperfect timing and non-ideal factional CFO compensation, a $0.4 \mathrm{~dB}$ degradation from the lower bound is observed in Fig. 5. This degradation from the theoretical lower bound is mainly due to the following two factors. Firstly, coarse timing position causes a loss of data and phase rotation after FFT operation. Secondly, residual fractional CFO also causes SNR loss, which can be quantified as [12]

$$
\mathrm{SNR}_{\text {loss }}(\mathrm{dB}) \leq 10 \cdot \log \left(\frac{1+0.5947 \cdot \mathrm{SNR} \cdot(\sin \pi \varepsilon)^{2}}{(\sin \pi \varepsilon / \pi \varepsilon)^{2}}\right),
$$

where $\varepsilon=f_{\text {frc }}-\hat{f}_{\text {frc }}$ is the normalised residual CFO after the fractional CFO compensation.

The signalling detection performances of the P1 symbol in DVB-T2 are also shown in Fig. 5 for comparison. Note that the S1 and S2 fields of the P1 symbol have different priorities while the 7-bit signalling of the proposed preamble has the same priority. The proposed signalling could achieve similar robustness as the S1 signalling, while outperforming the S2 signalling by about $0.5 \mathrm{~dB}$. 
TABLE I

CHANNEl PARAMETERS.

\begin{tabular}{c|cc|cc}
\hline \hline & \multicolumn{2}{|c|}{ Brazil-B } & \multicolumn{2}{c}{ GD-8 } \\
\hline $\begin{array}{c}\text { Echo } \\
\text { Index }\end{array}$ & $\begin{array}{c}\text { Delay } \\
(\mu s)\end{array}$ & $\begin{array}{c}\text { Power } \\
(d B)\end{array}$ & $\begin{array}{c}\text { delay } \\
(\mu s)\end{array}$ & $\begin{array}{c}\text { Power } \\
(d B)\end{array}$ \\
\hline 0 & 0.00 & 0.0 & -1.80 & -18.0 \\
1 & 0.30 & -12.0 & 0.00 & 0.0 \\
2 & 3.50 & -4.0 & 0.15 & -20.0 \\
3 & 4.40 & -7.0 & 1.80 & -20.0 \\
4 & 9.50 & -15.0 & 5.70 & -10.0 \\
5 & 12.70 & -22.0 & 30.00 & 0.0 \\
\hline \hline
\end{tabular}

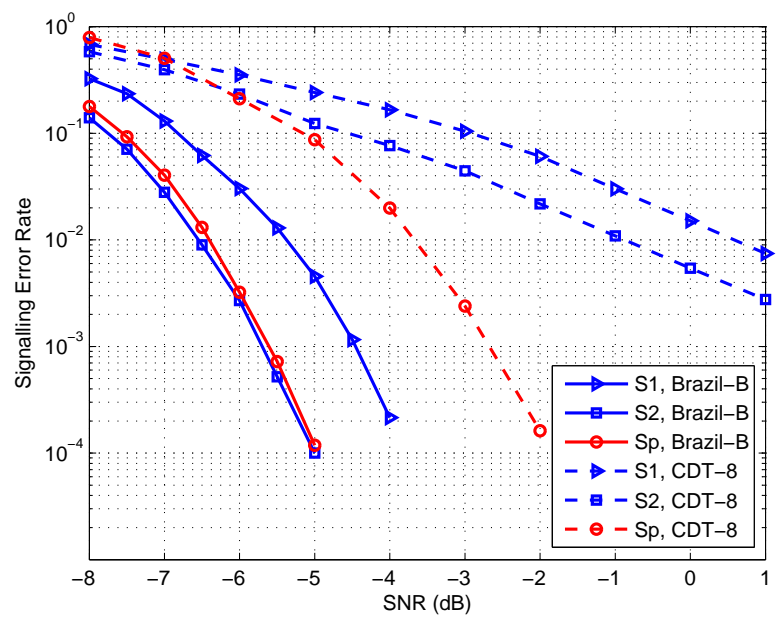

Fig. 6. Signalling error rate comparison of the $\mathrm{S} 1$ and $\mathrm{S} 2$ signalling in the $\mathrm{P} 1$ preamble as well as the proposed signalling (Sp), over the Brazil-B and CDT-8 frequency-selective fading channels.

\section{B. Simulations over Frequency-Selective Fading Channels}

A simulation study was then carried out to compare the signalling detection performance of the proposed preamble with that of the existing P1 symbol over frequency-selective fading channels. The Brazil DTV field test 2th channel model (Brazil-B) [4] and the China DTV test 8th channel model (CDT-8) [16] were adopted in the simulation investigation. The multi-path profiles of the both channels are listed in Table I. It should be noted that one $0 \mathrm{~dB}$ echo with a long time delay occurs in the CDT- 8 channel model, which will result in severely frequency-selective fading.

Fig. 6 depicts the simulation results for the S1 and S2 signallings of the P1 preamble as well as the proposed signalling (labelled as $\mathrm{Sp}$ in Fig. 6), respectively, over the Brazil-B and CDT- 8 channels. It can be seen from Fig. 6 that the proposed novel preamble design achieves a better SER performance than the existing P1 symbol design. To ensure an SER level of less than $10^{-2}$, for example, the proposed preamble design offers more than $3 \mathrm{~dB}$ SNR improvement in comparison with the P1 symbol for the CDT-8 channel. This is particularly significant, considering the fact that our proposed design imposes a lower complexity in signalling detection than the P1 preamble design.

\section{CONCLUSIONS}

We have proposed a novel preamble design, which exploits the variable distance between a pair of training sequences for OFDM transmission parameter signalling. Compared to the existing P1 symbol of the DVB-T2, the proposed preamble has a same high performance and robustness in timing and carrier frequency offset estimation while significantly reducing the signalling detection complexity at the receiver. Moreover, simulation results have demonstrated that the proposed novel preamble design achieves a better signalling detection performance than the existing P1 preamble design.

\section{ACKNOWLEDGMENTS}

This work was supported in part by Tsinghua University Initiative Scientific Research Program 20091081280 and in part by Standardization Administration of the People's Republic of China (SAC) with AQSIQ Project 200910244.

\section{REFERENCES}

[1] L. Hanzo, M. Münster, B. J. Choi and T. Keller, OFDM and MC-CDMA for Broadband Multi-User Communications, WLANs and Broadcasting. Chichester, U.K.: John Wiley \& Sons, 2003.

[2] H. Sari and G. Karam, "Orthogonal frequency division multiple access and its application to CATV networks," Eur. Trans. Commun., vol.45, pp.507-516, Nov. 1998.

[3] M. Takada and M. Saito, "Transmission system for ISDB-T," Proc. IEEE, vol.96, no.1, pp.251-256, Jan. 2006.

[4] J. Song, Z. X. Yang, L. Yang, K. Gong, C. Y. Pan, J. Wang and Y. S. Wu, "Technical review on Chinese digital terrestrial television broadcasting standard and measurements on some working modes," IEEE Trans. Broadcast., vol.53, no.1, pp.1-7, Mar. 2007.

[5] Frame Structure Channel Coding and Modulation for a Second Generation Digital Terrestrial Television Broadcasting System (DVB-T2). DVB Document A122, ETSI Std., Jun. 2008.

[6] IEEE Standard for Wireless LAN Medium Access Control (MAC) and Physical Layer (PHY) Specifications. IEEE 802.11, Nov. 1997.

[7] H. Ekstrom, A. Furuskar, J. Karlsson, M. Meyer, S. Parkvall, J. Torsner, and M. Wahlquist, "Technical solutions for the 3G long-term evolution," IEEE Commun. Mag., vol.44, no.3, pp.38-45, Mar. 2006.

[8] F. Khan, LTE for 4G Mobile Broadband: Air Interface Technologies and Performance. Cambridge, U.K.: Cambridge University Press, 2009.

[9] T. M. Schmidl and D. C. Cox, "Robust frequency and timing synchronization for OFDM," IEEE Trans. Commun., vol.45, no.12, pp.16131621, Dec. 1997.

[10] F. Tufvesson, M. Faulkner, and O. Edfors, "Time and frequency synchronization for OFDM using PN sequence preambles," in Proc. VTC'99. Fall, Sep. 1999, vol.4, pp.2203-2207,

[11] A. Viemann, A. Waadt, C. Spiegel, C. Kocks, A. Burnic, P. Jung, G. H. Bruck, J. Kim, J. Lim, and H.W. Lee, "Implementation-friendly synchronisation algorithm for DVB-T2," Electron. Lett., vol.46, no.4, pp.282-283, Feb. 2010

[12] P. H. Moose, "A technique for orthogonal frequency division multiplexing frequency offset correction," IEEE Trans. Commun., vol.42, no.10, pp.2908-2914, Oct. 1994.

[13] Implemention Guidelines for a Second Generation Digital Terrestrial Television Broadcasting System (DVB-T2). DVB Document A133, ETSI Std., Feb. 2009.

[14] T. May, H. Rohling, and O. Edfors, "Reducing the peak-to-average power ratio in OFDM radio transmission systems," in Proc. VTC'98Spring, May 1998, vol.3, pp.2474-2478.

[15] H. G. Proakis, Digital Communications (Third edition). McGraw-Hill Book Co., 1995.

[16] F. Yang, J. T. Wang, J. Wang, J. Song, and Z. X. Yang, "Novel channel estimation method based on PN sequence reconstruction for Chinese DTTB system," IEEE Trans. Consum. Electron., vol. 54, no. 4, pp. $1583-$ 1589, Nov. 2008 